\title{
Workplace based assessment (WBA) - an introduction
}

\author{
Gunatilake S B ${ }^{1}$ \\ Journal of the Ceylon College of Physicians, 2014, 45, 57-58
}

College members as trainers of the Post Graduate Institute of Medicine play a vital role in training and assessing future physicians. The training and assessment methods have evolved and keep changing to suit the needs of the day. The Medicine training programme of the PGIM in keeping with this trend will be introducing changes to suit the current and future developments. Aiming to update the members on these changes the Journal will be publishing a series of articles by trainers to satisfy this need and this is the first article of the series.

The integrated assessment systems used in current post graduate training schemes comprise a mixture of workplace-based assessments and knowledge-based assessments.

Workplace Based Assessment (WBA) is the assessment of competence based on what a trainee actually does in the workplace. The main aim of WBA is to aid learning (Assessment for learning) by providing trainees with constructive feedback. Trainees can use the same methodology to assess themselves (Reflective practice). The assessments help the Supervisor to chart a Trainee's progress during a placement. WBA should be trainee-led; the trainee choosing the method, timing, activity and under the guidance of the Supervisor according to the Learning outcomes laid out in the Prospectus. Trainees are encouraged to use as many different assessments and assessors as possible, as this improves reliability.

WBAs in many forms will be introduced in the new curricular changes to the MD Medicine programme to be implemented in 2015. In such a situation the trainers need to be aware of, be knowledgeable and capable in implementing these methods to assessing the trainees.

\section{Methods of WBA are}

- Mini-Clinical Evaluation Exercise (mini-CEX)

- Direct Observation of Procedural Skills (DOPS)

- Multi-Source Feedback (MSF)

\footnotetext{
${ }^{1}$ Professor of Medicine, Faculty of Medical Sciences University of Sri Jayewardenepura, Sri Lanka.
}

- Case-Based Discussions (CbD)

- Acute Care Assessment Tool (ACAT)

\section{Mini-Clinical Evaluation Exercise (mini-CEX)}

This tool evaluates a clinical encounter with a patient to provide an indication of competence in skills essential for good clinical care such as history taking, examination, clinical reasoning, communication and professionalism. The trainee receives immediate feedback to aid learning. This can be used at any time and in any setting when there is a trainee and patient interaction and an assessor is available. This is expected to last about 20 minutes.

\section{Direct Observation of Procedural Skills (DOPS)}

DOPS is an assessment tool designed to evaluate the performance of a trainee in undertaking a practical procedure, against a structured checklist. The trainee receives immediate feedback to identify strengths and areas for development. Portfolio will give a list of procedures suitable to be assessed at DOPS. Areas assessed will include understanding of indications, anatomy and technique, pre-procedure preparation, ability, post-procedure management and professionalism.

\section{Multi-source feedback (MSF)}

This tool is a method of assessing generic skills such as communication, leadership, team working, reliability etc, across the domains of Good Medical Practice. This provides objective systematic collection and feedback of performance data on a trainee, derived from a number of colleagues. 'Raters' are individuals with whom the trainee works, and includes doctors, administration staff, and other allied professionals. The trainee will not see the individual responses by raters. Feedback is given to the trainee by the Supervisor.

\section{Case-based Discussion (CbD)}

The CbD assesses the performance of a trainee in their management of a patient to provide an indication of competence in areas such as clinical reasoning, decision-making and application of medical knowledge in relation to patient care. It also serves as a method to document conversations about, and presentations 
of, cases by trainees. The CbD should focus on a written record (such as written case notes, diagnosis card, and discharge summary) of a patient they have recently seen. Cases for discussion are chosen by the assessor. This is expected to last 30 minutes. Following areas are assessed - record keeping, history taking, clinical findings and interpretation, management plan, ethical issues, and follow up.

\section{Acute Care Assessment Tool (ACAT)}

The ACAT is designed to assess and facilitate feedback on a trainee's performance during their practice on the casualty admissions. Any trainer who has been responsible for the supervision of the casualty admissions can be the assessor for an ACAT. This is expected to last about 15 minutes. 